\title{
A Bayesian framework for the development of belief-desire reasoning: Estimating inhibitory power
}

\author{
Lu Wang $^{1} \cdot$ Pernille Hemmer $^{1} \cdot$ Alan M. Leslie ${ }^{1}$
}

Published online: 20 July 2018

(C) Psychonomic Society, Inc. 2018

\begin{abstract}
A robust empirical finding in theory-of-mind (ToM) reasoning, as measured by standard false-belief tasks, is that children four years old or older succeed whereas three-year-olds typically fail in predicting a person's behavior based on an attributed false belief. Nevertheless, when the child's own belief is undermined by increasing their subjective uncertainty about the truth, as introduced in low-demand false-belief tasks, three-year-olds can better appreciate another person's false belief. Inhibition is believed to play a critical role in such developmental patterns. Within a Bayesian framework, using meta-data, we present the first computational implementation of inhibition, as specified by the Theory of Mind Mechanism (ToMM) model, to account for both the developmental shift from three to four years of age and the change in children's performances between high-demand and lowdemand false-belief tasks. A Bayesian framework enables us to evaluate the predictive power of the model and infer the underlying psychological parameters. Together with behavioral evidence, we discuss the critical role of inhibitory control, as specified by ToMM, in children's theory-of-mind development.
\end{abstract}

Keywords Theory of mind $\cdot$ Rationality $\cdot$ Computational model $\cdot$ Bayesian $\cdot$ Inhibition

\section{Introduction}

Understanding the actions of others is critical for our social interactions. We do this by attributing mental states, such as beliefs and desires, to others. The cognitive basis of this skill, theory of mind (ToM), includes two main inferential processes. On the one hand, we can infer a person's belief on the assumption that beliefs are determined by access to the environment. Together with information about the person's desire, and the rational assumption that a person's action is the most efficient way to satisfy his/her desire in light of his/her belief, we can predict what the person will most likely do (Dennett, 1989). On the other hand, assuming the principle of rational behavior, we can infer the mental states of others given observed behavior by reversing the causal connections between belief, desire, and action (e.g., Baker, Saxe, \& Tenenbaum, 2009; Baker, Goodman, \& Tenenbaum, 2008; Baker, Saxe, \& Tenenbaum, 2011; Richardson, Baker, Tenenbaum, \&

Alan M. Leslie

aleslie@ ruccs.rutgers.edu

1 Department of Psychology and Center for Cognitive Science, Rutgers University, Rutgers, NJ, USA
Saxe, 2012). Here we investigate how these two rational assumptions interact with a third assumption that beliefs ought to be, and typically are, true (Dennett, 1989; Leslie, 1994), and how these rational assumptions in computing mental states manifest in theory-of-mind development.

Theory of mind has been extensively studied, especially in young children. Children's ToM reasoning is commonly assessed using the "Sally and Anne" false-belief task (Baron-Cohen, Leslie, \& Frith, 1985). In this task, one actor, Sally, hides an object in location A and then departs. A second actor, Anne, moves this object and hides it in location B. The child is asked to predict where Sally will search for the object when she returns. This well-established paradigm has shown that the majority of typically developing children over four years of age pass this task by predicting that Sally will search location A, where she still believes the object to be. However, three-year-olds typically fail and predict Sally will search location $B$, where the object really is.

While the false-belief task has been instrumental in establishing the developmental trajectory of ToM, there is also empirical evidence showing that even younger children are able to attribute a false belief correctly. For example, Setoh, Scott, and Baillargeon, (2016) found that when the target object in the "Sally and Anne" task is moved to an unknown location after 
"Anne" moved it to location B, so that the child has no certain belief about its final location, two-and-half-year-olds are better at attributing false beliefs. In other words, moving the object to an unknown location makes children younger than four years of age more likely to appreciate others' false belief.

Several other studies lend support to such improvements in children's ToM reasoning. For example, when children were only told about the target object's whereabouts, but did not see the real location (Carlson \& Moses, 2001; Zaitchik, 1991); when the actor announces her belief about the object's location while the child sees both locations are in fact empty (Bartsch, 1996); when a character makes his "Plasticine" molding material into a hat, but the Plasticine is reshaped into an apple and relocated while he is away (Kikuno, Mitchell, \& Ziegler, 2007). In all of these scenarios, the child's confidence in his/her own belief about the specific location/identity of the target object is undermined, producing what we term a "lowdemand" (LD) task (Wang \& Leslie, 2016). The increased uncertainty/reduced confidence in their own belief facilitates the attribution of a false belief in LD tasks relative to "highdemand" (HD) tasks. Standard false-belief tasks, in which the child is certain about the target's final whereabouts, make it more difficult for the child to overcome the tendency to attribute her own belief to the actor.

The complex findings of the developmental trajectory from three to four years of age, and the facilitation of false belief for three-year-olds in low-demand tasks, provide a particularly challenging pattern of data for understanding theory-of-mind reasoning.

Advances in understanding the developmental transition in ToM performance can be made via computational models. For example, young children may indeed understand that people have beliefs, but become better at inhibiting a bias to attribute their own belief in a false-belief scenario (Leslie, German, \& Polizzi, 2005). Recently, a connectionist neural network implementation of the false-belief task (Berthiaume, Shultz, \& Onishi, 2013) suggested that transition in ToM performance is due to increases in processing resources for inhibiting own true belief. In both models, younger children fail the tasks because processing demands are beyond the developmental stage of the child.

These ideas are consistent with theoretical accounts emphasizing the critical role of inhibitory control in ToM development. For example, Russell (Russell, 1996), and Carlson and her colleagues (e.g. Carlson, Moses, \& Hix, 1998) suggested that inhibition (or executive function) enables children to consider evidence about others' mental states, and hence promotes the development of theory of mind. A more detailed theory, known as the Theory of Mind Mechanism (ToMM; Leslie, 1994; Leslie, German, \& Polizzi, 2005; Leslie \& Polizzi, 1998; Leslie \& Thaiss, 1992), posits that from the outset children have an adult-like rational competence in theory-of-mind reasoning but lack inhibitory power. To succeed in predicting Sally's behavior, children have to inhibit their own true belief about the object's location. The inhibitory power to overcome one's own perspective develops slowly from three to four years of age (Beck, Schaefer, Pang \& Carlson, 2011; Carlson, 2005; Diamond, Carlson \& Beck, 2005); individual false-belief performance typically remains unstable for long periods (S. Baker, Leslie, Gallistel, \& Hood, 2016), giving rise to the familiar developmental pattern only through group averaging.

We aimed to implement the first computational model using a Bayesian framework for the role of inhibition in executing ToM capacity, and use it to model the developmental shift from three to four years of age. Our goal is to then extend this account to the effect of task demand in children's beliefdesire reasoning - specifically, that three-year-olds are more likely to understand another's false belief when they are less certain about their own true belief. Such variation in children's performance has never been formally modeled before. In particular, we adopted the ToMM view of theory-of-mind development by formalizing the rational processes in theory-ofmind reasoning using a Bayesian framework.

Our reasons for implementing ToMM are threefold. First, ToMM is by far the most computationally specified account showing how inhibitory control might be incorporated in ToM reasoning. Second, while there are other theoretical views explaining how ToM develops in preschoolers, such as the "theory theory" (for a review, see Wellman, Cross, \& Watson, 2001), these are not well specified for task demand. For example, theory-theory posits that the rational (adult) model for belief-desire reasoning is not present until the age four shift and must be absent earlier and certainly absent in infancy. Although theory-theory makes similar predictions to ToMM for the developmental trajectory from three to four years (but see S. Baker et al., 2016), it does not make specific predictions about the facilitatory effect of LD tasks in younger children. By contrast, because ToMM assumes that younger children possess the same rational model as adults in theory-of-mind reasoning, it also predicts that changes in task demand can improve performance, even for children with lower inhibitory power. For example, in the LD false-belief task, the child herself does not have a competing belief about the specific location of the target object (Bartsch 1996; Carpenter et al., 2002; Kikuno et al., 2007; Setoh, Scott, \& Baillargeon, 2016), making it easier to inhibit. This in turn makes it more likely the child will attribute a false belief (Wang \& Leslie, 2016). As such, the ToMM provides a unified account for both the developmental trajectory and the facilitatory effect of low demand on theoryof-mind reasoning.

Finally, although it remains controversial how the concepts of mental states emerge in children, recent experimental findings suggest an early understanding of false belief, even in infancy (e.g., Onishi \& Baillargeon, 2005; Southgate, Senju, \& Csibra, 2007; for reviews, see Baillargeon, Scott, \& He, 
2010; Scott \& Baillargeon, 2017). Although such findings support long-standing predictions of the ToMM model (see especially Leslie, 1994), our focus here is on modeling the three- to four-year shift, which we model as a shift in performance without assuming any change in conceptual repertoire.

The plan for this paper is as follows. We first describe the details of the ToMM, and define the formal model. We will then test the model on meta-data for the standard false-belief task (i.e., high demand). Finally, we will introduce the formal model for the low-demand task, and test it on meta-data.

\section{ToMM and the formal model}

\section{ToMM + selection processing}

Given that adults' belief-desire reasoning follows a rational model (Dennett, 1989), a parsimonious account of ToM in children would suggest that three- and four-year-olds are using the same rational model for belief-desire reasoning. This basic assumption of rationality throughout development underlies the ToMM account. According to ToMM, the failure of younger children in the standard false-belief task is hypothesized to be caused by their immature inhibitory abilities (Leslie \& Thaiss, 1992; Roth \& Leslie, 1998; Russell, Mauthner, Sharpe, \& Tidswell, 1991; Scholl \& Leslie, 2001; cf., Carlson, Moses, \& Hix, 1998). Specifically, ToM is a spontaneous process supported by a domain-specific module, the theory-of-mind mechanism (ToMM), which mandatorily processes information about people's mental states whenever one attends to an agent (Leslie, 1987, 1994).

In a given scenario, the ToMM computes all plausible contents for belief attribution, among which is a "true belief," derived from one's own knowledge of the scene. In the false-belief scenario, another candidate, a "false belief" will also be calculated derived from the other person's visual access (or more generally, informational access) to the scenario. The candidates for belief attribution will then be reviewed, and the true belief (TB) content is a priori more likely to be selected, unless there is evidence to the contrary. However, in the false-belief (FB) scenario, the a priori likelihood of selecting TB (TB prior) will produce a bias that needs to be inhibited for the false-belief content to be selected. The addition of Selection Processing (SP) to the ToMM (ToMM + SP) instantiates this inhibition of the TB prior (Leslie, German, \& Polizzi, 2005). According to this account, younger children possess the competent model of ToM but encounter difficulties with applying this competence when the required inhibitory power is not available, i.e., for the standard task before the age of four, and for certain more demanding tasks before the age of six or beyond (Friedman \& Leslie, 2004a, b; Leslie, Friedman, \& German, 2004; Leslie et al., 2005).
Consistent with this hypothesis, in experimental tasks that lower the demands on SP by balancing the salience of the true and false representations, three-year-old children are more likely to understand false belief (Bartsch, 1996; Carpenter, Call, \& Tomasello, 2002; Kikuno, Mitchell, \& Siegler, 2007; Koós, Gergely, Csibra, \& Bíró, 1997; Surian \& Leslie, 1999; Wellman \& Bartsch, 1988; Zaitchik, 1991). In contrast, when the demands of SP are increased, even four-year-olds fail to demonstrate false-belief understanding (Cassidy, 1998; Friedman \& Leslie, 2004a \& b; Leslie et al., 2005; Leslie \& Polizzi, 1998). Furthermore, the role of inhibitory control in belief-desire reasoning is not exclusive to early development. Adults, whose ToM competence is well established, still require sufficient inhibitory ability to succeed in false-belief tasks (Apperly, Samson, Chiavarino, \& Humphreys, 2004; German \& Hehman, 2006; Hartwright, Apperly, \& Hansen, 2012; McKinnon \& Moscovitch, 2007; Samson, Apperly, Kathirgamanathan, \& Humphreys, 2005; Saxe, Schulz, \& Jiang, 2006; van der Meer, Groenewold, Nolen, Pijnenborg, \& Aleman, 2011). For example, German and Hehman (2006) found that adults performed worse when the false-belief tasks' processing demands were increased. Furthermore, they found that such effects were greater in elderly individuals whose inhibitory abilities had declined due to cognitive aging. In a study that allowed neural and behavioral probing of ToMMSP fractionation, LeBouc et al. (2012) found that in Alzheimer patients lower activity in the temporoparietal junction (TPJ) was associated with poorer performance in inferring others' false beliefs, whereas in behavioral variant frontotemporal dementia lower activity in right medial frontal gyrus was associated with difficulty inhibiting own- (true-) belief.

In summary, the ToMM+SP model assumes that adults and children use the same rational model for ToM reasoning. What underlies the developmental shift from three to four years of age in the false-belief task is an increasing power of inhibition to overcome the TB prior. Three core elements of belief attribution can thus be identified according to ToMM:

1. All plausible candidates for belief attribution. Typically, only a true belief that reflects or is consistent with one's own perspective is present; but in false-belief scenarios, when one is aware of the other person's lack of information access, a belief consistent with the outdated information would be calculated. It might be counterintuitive to imagine that a true belief that is no longer appropriate in false-belief scenarios might exist. However, empirical evidence has suggested that in false-belief tasks, both a truebelief and a false-belief candidate are available, and they compete to be selected (Kikuno et al., 2007 Wang \& Leslie, 2016).

2. A true belief prior. The true belief that is consistent with one's own perspective is a priori more likely to be selected. For this reason, a true-belief prior can be regarded as 
an own-belief prior. Note that throughout we use "confidence" and "certainty" interchangeably as subjective values that are modeled as "noise."

3. Given 1 and 2, in order to select the false-belief candidate, inhibition is required to overcome the $a$ priori of selecting the true belief. Consequently, changes in the inhibitory power would give rise to different performances in falsebelief tasks.

\section{ToMM+SP model}

In the Sally-and-Anne false-belief task, Sally puts her toy in location $\mathrm{A}$ and while she is away the toy is relocated to location B. The goal of the child is to infer the belief of Sally, which differs from the child's own belief, based on their observation of the true state of the world and Sally's perceptual access to it. The child's inference process is conditioned on two variables of interest: whether the object has changed location (true state of the World) and whether Sally has visual access to the change (View). Then, based on the child's inference of Sally's belief and her desire, the child will predict Sally's action when she returns (Action). Assuming the theoretical arguments outlined above, the next section illustrates the computational model implementing the ToMM account of the developmental shift of children's action prediction in a standard false-belief task.

Following convention, we adopt graphic models as a way to visualize structure. In the graphic model, circular nodes represent all variables of interest. Nodes for observed variables are shaded while nodes for latent variables are unshaded. Further, stochastic variables are differentiated with a single border and deterministic variables with double borders. Arrows indicate conditional dependencies.

For the child to infer the unobserved variable of Sally's belief, ToMM computes all plausible belief contents, with a strong prior propensity (TB prior) to select a "true-belief" content derived from the child's own knowledge of the world $(W)$. In the false-belief scenario, the false-belief content is calculated based on Sally's history of visual access $(V)$ to the change. Importantly, inhibitory power $(I)$ over the true-belief (TB) content develops from three to four years of age, making it more likely for four-year-olds to select the false-belief $(F B)$ content. The selected belief $(B)$, together with information of Sally's desire $(D)$, enables the child to predict Sally's action (A). The implementation of the ToMM + SP model assumes that all children within their own age group (three-year-olds or four-year-olds) possess similar inhibitory power (Fig. 1).

In the standard false-belief task, Sally wants her toy back, and she will go to the location where she believes the toy to be. In this case, Desire $(D)$ is assumed to have a large prior probability, $1-\varepsilon$, favoring the target object, in which $\varepsilon$ represents all possibilities that Sally may change her interest. Therefore, we use an asymmetric beta prior on $\varepsilon$, with $\varepsilon \sim \operatorname{Beta}(1,10)$, indicating that Sally is unlikely to change her desire without information to the contrary. ${ }^{1}$

The true belief content one attributes to Sally $(T B)$ is determined by one's own knowledge of the world, with probability $W-\gamma_{T B}$, where $W=1$ or 0 , indicating change in the world or no change, and $\gamma_{T B}$ represents variations in how one may represent the world incorrectly. The false-belief content $(F B)$ is determined by Sally's visual access to the world ( 0 , or no access, in a false-belief scenario), with the uncertainty about $F B$ given by the absolute value of $\left(W \cdot V-\gamma_{F B}\right)$. Again, we assume asymmetric beta priors on $\gamma_{T B}$ and $\gamma_{F B}$, with $\gamma_{T B} \sim$ Beta $(1,20)$ and $\gamma_{F B} \sim \operatorname{Beta}(1,20)$, indicating that one has an accurate representation of the world and others visual access.

Under the assumption of ToMM + SP, to succeed in the standard false-belief task, the child has to deploy sufficient inhibition $(I)$ to overcome the TB prior. We assume that $I$ follows the Beta distribution with $I \sim \operatorname{Beta}(\alpha, \beta)$, where $\alpha \sim$ Uniform $(0,100)$ and $\beta \sim$ Uniform $(0,100)$, indicating no prior assumptions about the shape of the beta distribution of $I$ and to allow for a complete estimation of $I$ from the observed data.

Therefore, the attributed belief is the most active belief between $F B$ and $T B$ with inhibition. That is, the probability of $B$ is the larger one between the probability of the true belief with an inhibition $\left(W-\gamma_{T B}-I\right)$ and the probability of the false belief (the absolute value of $W \cdot V-\gamma_{F B}$ ). After the selection, $B$ $\sim$ Binomial $(1, \max (F B, T B-I))$, the child attributes either the belief that there is no change $(B=0)$ or the belief that there is a change $(B=1)$ to the agent. Attributed belief coupled with desire determines the child's action prediction $(A)$. In particular, when the attributed desire is to retrieve the object $(D=1)$, the child's prediction follows the attributed belief $(B)$ and $A \sim$ Binomial $(1, B)$; when the attributed desire is ambiguous $(D=$ 0 ), the child's action prediction is random and $A \sim$ Binomial $(1,0.5)$. Table 1 gives the conditional probabilities amongst all variables.

\section{Bayesian inferences and model predictions}

\section{The data set}

To infer the underlying psychological parameters in our implementations of the ToMM model, we extracted data from the extensive existing literature. The performance of three- to four-year-olds in false-belief tasks has been well documented and the findings are uncontroversial and highly consistent (except for slight variations, see below). Therefore, we surveyed the literature and summarized children's performances in a standard verbal false-belief task as the inputs to the

\footnotetext{
${ }^{1}$ Goodman et al. (2006) provided an implementation of theory-theory that enumerates these fundamental relationships, and provides the distributional assumptions. We adopt these same distributional assumptions.
} 


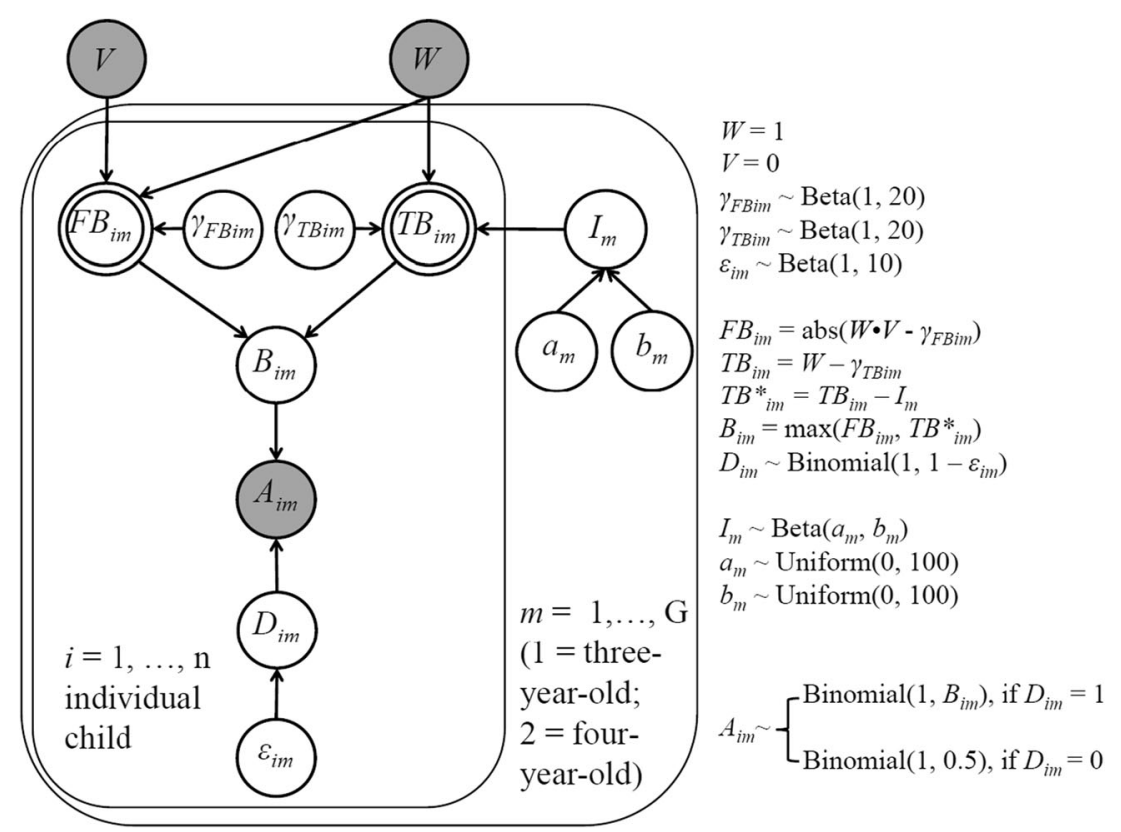

Fig. 1 The graphic model for ToMM + SP. W: world; $F B$ : false belief; $T B$ : true belief derived from one's own knowledge; $D$ : desire; $A$ : action; $V$ : visual access; $I$ : inhibition

models. Specifically, we consider data from the (change-oflocation) Sally-and-Anne task. There are a number of other popular tasks for assessing false belief, e.g., the unexpectedcontents or deceptive-appearance task (Gopnik \& Astington, 1988; Perner, Leekam, \& Wimmer, 1987; Wimmer \& Hartl, 1991). However, there are some variations in reported performance depending on whose belief state was probed (another person's belief or one's own belief at an earlier moment), whether purposeful deceptions were used (Chandler, Fritz, \& Hala, 1989), whether there were manipulations over the salience of the true-belief or the false-belief content (Bartsch, 1996; Carpenter, Call \& Tomasello, 2002; Kikuno, Mitchell, \& Ziegler, 2007; Mitchell \& Lacohée, 1991; Wellman \& Bartsch, 1988), and on children's cultural background (Callaghan et al., 2005; Liu, Wellman, Tardif, \& Sabbagh, 2008; Wellman et al., 2001). We therefore chose to focus strictly on the Sally-and-Anne task. Nevertheless, findings across a wide variety of false-belief tasks are highly consistent and replicable (Wellman et al., 2001), and some inhibitory control is probably necessary in all the versions. With slight modification, our implementation can be applied to other standard scenarios.

We constrained our search to studies that used the standard Sally-and-Anne task that were conducted with children in Western cultures, without deception or salience manipulations. Furthermore, tasks in which the findings were based on children's performances in more than one false-belief task (that is, performances in the individual tasks were not reported) or in which the findings were grouped together for children of different ages were excluded, unless children's performances in each false-belief task and for each age group could be recovered.

We selected a total of 17 studies from 15 publications, including performances from 398 three-year-olds and 332 four-year-olds. This list was not intended to be exhaustive, but representative at the time of model implementation, according to the selection criteria outlined above. See Table 2 for the list of selected publications and studies. Overall, threeyear-olds have a much lower success rate (mean Observed: 0.27 , s.d. $=0.109$ ) relative to four-year-olds (mean Observed: 0.63 , s.d. $=0.149)$ in all of the selected studies.

Table 1 Conditional probability table for ToMM model

\begin{tabular}{llllllll}
\hline $\mathrm{P}(A=1 \mid B, D)$ & $B$ & $D$ & $\mathrm{P}\left(B=1 \mid F B, T B, I, W, V, \gamma_{T B}, \gamma_{F B}\right)$ & $F B$ & $W$ & $V$ & $T B$ \\
\hline 1 & 1 & 1 & $\gamma$ & $\gamma_{F B}$ & 0 & 0 & $\gamma_{T B}$ \\
0 & 0 & 1 & $\gamma$ & $\gamma_{F B}$ & 0 & 1 & $\gamma_{T B}$ \\
0.5 & 1 & 0 & $1-\gamma$ & $1-\gamma_{F B}$ & 1 & 1 & $1-\gamma_{T B}$ \\
0.5 & 0 & 0 & $1-\gamma_{T B}-I$ & $\gamma_{F B}$ & 1 & 0 & $1-\gamma_{T B}$ \\
\hline
\end{tabular}

Note: $\mathbf{P}(\boldsymbol{A}=\mathbf{1} \mid \boldsymbol{B}, \boldsymbol{D})$ is the conditional probability of Action $=1$ given the specific combination of Belief and Desire; $\mathbf{P}\left(\boldsymbol{B}=\mathbf{1} \mid \boldsymbol{F B}, \boldsymbol{T B}, \boldsymbol{I}, \boldsymbol{W}, \boldsymbol{V}, \gamma_{\boldsymbol{T B}}, \gamma_{\boldsymbol{F B}}\right)$ is the conditional probability of attributing a Belief $=1(B \sim \operatorname{Binomial}(1, \max (F B, T B-I))$, $\max (F B, T B-I)$ is the probability of $\mathrm{B}=1)$ given the false belief, true belief, available inhibotory power, the real status of the world, the agent's visual access, and the noises of each belief 
Table 2 Selected publications using standard Sally-and-Anne task measuring children's false-belief reasoning

\begin{tabular}{|c|c|c|c|}
\hline Publication & Study/condition* & Age & Number of pass \\
\hline Bartsch, 1996 & Study $1, \mathrm{XO}$ and $\mathrm{XY}$ conditions & 3 & 4 out of 18 \\
\hline \multirow[t]{2}{*}{ Callaghan, et al., 2005} & \multirow[t]{2}{*}{ Canadian participants only } & 3 & 2 out of 13 \\
\hline & & 4 & 9 out of 17 \\
\hline \multirow[t]{2}{*}{ Carlson, Moses, 2001} & \multirow[t]{2}{*}{ Standard unexpected location } & 3 & 6 out of 62 \\
\hline & & 4 & 22 out of 45 \\
\hline \multirow[t]{2}{*}{ Clements, Rustin, McCalllum, 2000} & \multirow[t]{2}{*}{ Pre-test } & 3 & 18 out of 61 \\
\hline & & 4 & 20 out of 30 \\
\hline \multirow[t]{2}{*}{ Hickling, Wellman, Gottfried, 1997} & \multirow[t]{2}{*}{ Study 1} & 3 & 5 out of 18 \\
\hline & & 4 & 13 out of 16 \\
\hline \multirow[t]{2}{*}{ Kikuno, Mitchell \& Ziegler (2007) } & \multirow[t]{2}{*}{ Study 3 , discriminative condition } & 3 & 10 out of 29 \\
\hline & & 4 & 16 out of 29 \\
\hline Matsui, Rakoczy, Miura, \& Tomasello, 2009 & Study 1 & 3 & 5 out of 24 \\
\hline Peterson \& Siegal, 1999 & Change-location task, typical-developing children & 4 & 18 out of 21 \\
\hline Riggs, Peterson, Robinson, \& Mitchell, 1998 & Study 2 & 4 & 12 out of 28 \\
\hline Riggs \& Simpson, 2005 & Only those who passed memory control questions & 3 & 13 out of 36 \\
\hline \multirow[t]{4}{*}{ Robinson, Mitchell, 1995} & \multirow[t]{2}{*}{ Study 1} & 3 & 6 out of 20 \\
\hline & & 4 & 6 out of 13 \\
\hline & \multirow[t]{2}{*}{ Study 5} & 3 & 4 out of 11 \\
\hline & & 4 & 5 out of 9 \\
\hline Sabbagh, Bowman, Evriaire, \& Ito, 2009 & False belief: location & 4 & 53 out of 78 \\
\hline \multirow[t]{2}{*}{ Surian \& Leslie, 1999} & Study 1 & 3 & 6 out of 20 \\
\hline & Study 2 & 3 & 13 out of 40 \\
\hline \multirow[t]{2}{*}{ Wimmer, Weichbold, 1994} & \multirow{2}{*}{$\begin{array}{l}\text { Unique version condition, using action } \\
\text { prediction question }\end{array}$} & 3 & 1 out of 14 \\
\hline & & 4 & 9 out of 14 \\
\hline \multirow[t]{2}{*}{ Zaitchik, 1991} & \multirow[t]{2}{*}{ Seen \& standard condition } & 3 & 14 out of 32 \\
\hline & & 4 & 27 out of 32 \\
\hline \multirow[t]{2}{*}{ Summary } & & 3 & 107 out of 398 \\
\hline & & 4 & 210 out of 332 \\
\hline
\end{tabular}

*Indicating which study/condition in a given publication was selected for our data set if there were multiple studies/conditions presented

\section{Results}

The model was implemented using WinBUGS (Lunn, Thomas, Best, \& Spiegelhalter, 2000; Ntzoufras, 2009). Our results are based on drawing 3,000 samples from three separate chains with 100 burn-in period for each of the models. Convergence of the chains was assessed using the $\hat{R}$ statistic (Brooks and Gelman, 1998).

According to the Bayesian network in Fig. 1, children's action prediction $(A)$ is conditioned on attributed belief and desire $(B, D)$, in which $B$ is further determined by the selection between false belief $(F B)$ and true belief $(T B)$ with inhibition $(I)$. Therefore, knowing children's action prediction, we can reverse the causal chain, and infer the cognitive parameters $(I)$ that give rise to such action prediction in children of different ages. Figure 2 shows the posterior distribution of the inhibition parameter for each age group. It is clear that the posterior distribution of the inhibition parameter changes as a function of age group. The mean inhibitory power for three-year-olds is 0.20 (s.d. $=0.014,95 \%$ credible region is $0.172 \sim 0.228$ ), while the mean inhibitory power for four-year-olds is 0.60 (s.d. = $0.016,95 \%$ credible region is $0.563 \sim 0.627$ ), indicating that older children have a greater inhibitory power. It also appears that four-year-olds do not possess full inhibitory power, which means that they may still fail at the task. This developmental shift from three- to four-year-olds in the ability to inhibit the $a$ priori preferred true belief based on the child's own observations of the world is predicted by ToMM+SP.

Figure 3 shows the posterior predictives for action prediction from the model. The posterior predictives are predictions for future - yet to be observed - data based on the parameter values inferred from the observed data. One can think of this as how a child with these parameter settings in his/her cognitive model is likely to perform on this task the next time he/she is tested. The mean posterior predictives from the model are shown in black squares on the bar plot of the observed data. 


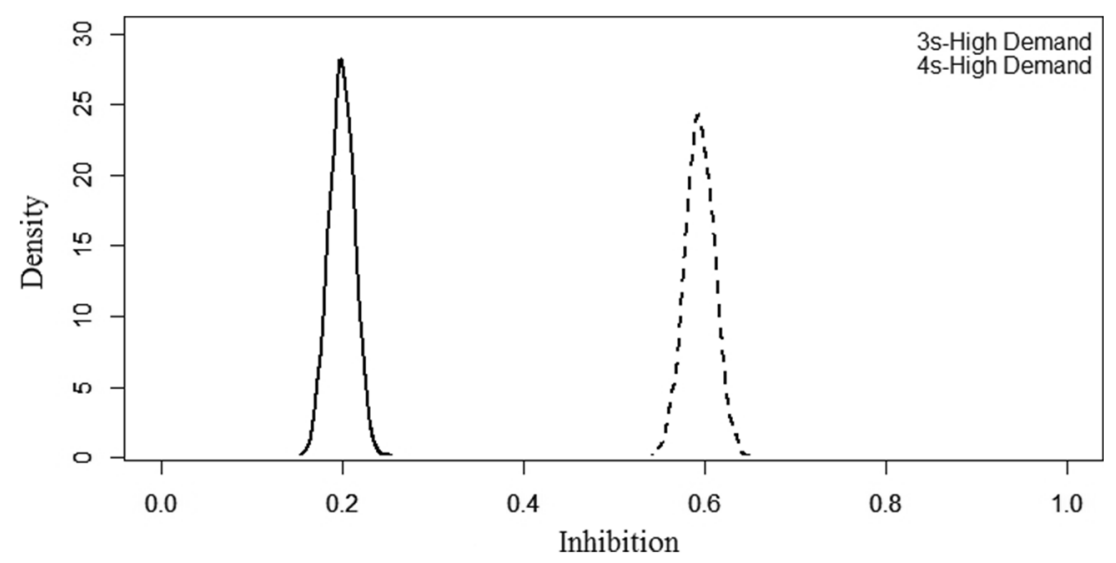

Fig. 2 Posterior distribution of inhibition (I), for each age group

The posterior predictive distribution is shown in the left panel. It is clear that the model captures the observed success rate in action predictions for each of the two age groups. Specifically, the model captures the trend that three-year-olds have a much lower success rate compared to four-year-olds (mean posterior predictives were 0.28 (s.d. $=0.068)$ and 0.62 (s.d. $=0.072$ ) for three-and four-year-olds, respectively).

\section{Discussion}

We implemented the role of inhibition in ToM reasoning, as framed by ToMM+SP, and evaluated the model's predictive power. Overall, it provides excellent qualitative fits for children's performances in the standard false-belief task, and for the developmental shift between three- and four-year-olds.
Specifically, three core elements of ToMM+SP are explicitly implemented in a Bayesian framework, and all are required to account for the observed behavioral patterns.

1. ToMM routinely and synchronously computes two possible contents as hypotheses about the agent's belief: one is based on (one's own) on-going and updating representation of the scenario or "world," the true-belief; and the other is based on the agent's history of visual (or other informational) access to that world. If only one candidate is available for belief attribution, one would either expect no understanding of others' outdated belief (only the true belief candidate), or no error in belief attribution once children start to appreciate a false belief. Either case is implausible given the empirical literature.

2. The initial preference in the ToMM-SP model is a prior derived from one's own perspective, a representation of

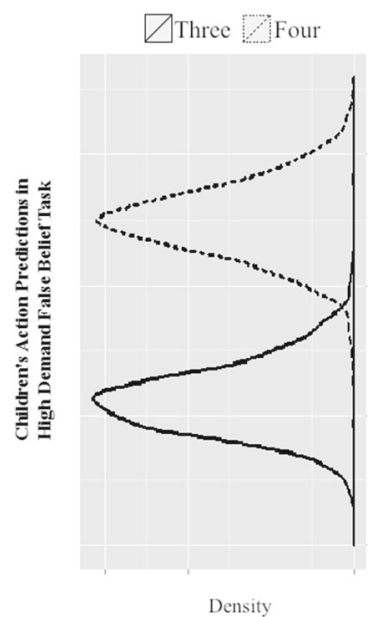

Fig. 3 Mean posterior predictives for action prediction in relation to the observed data (right panel) and the density of posterior predictives for action prediction (left panel) as a function of age group given the

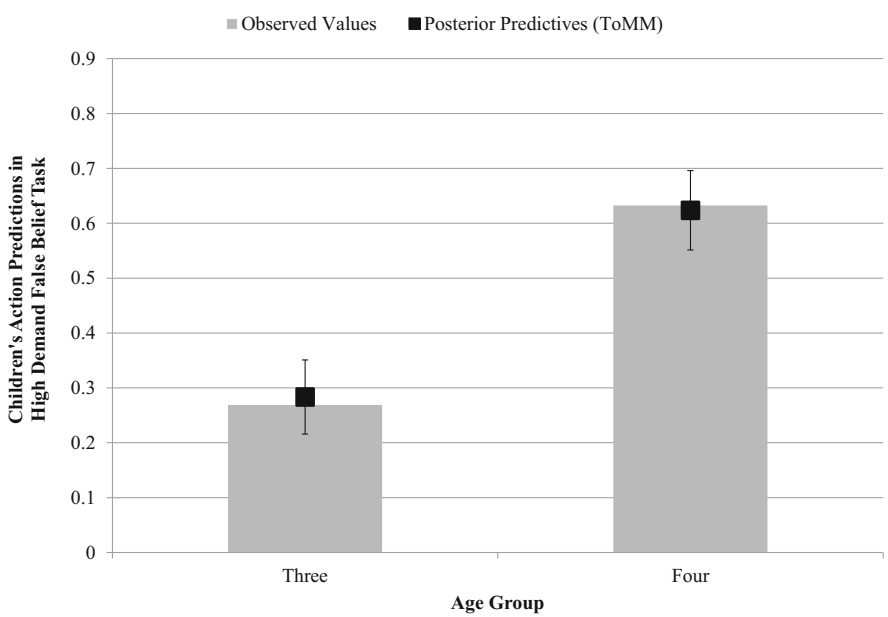

ToMM+SP model. Error bars represent the standard error of the posterior predictive distributions 
the world. The true-belief prior is part of ToMM's mode of operation, is a rational prior because people's beliefs typically are true, and reflects properties of ToM competence. If true belief and false belief are equally likely to be selected, one would expect a 50-50 chance of success in children with limited inhibitory power - again, this is not compatible with the established developmental trajectory of ToM reasoning observed in preschoolers. Furthermore, the prior of choosing true belief still operates in older children and adults, which longs for the third element, inhibition, for a successful operation of ToM reasoning.

3. A failure in false-belief reasoning would occur unless the true-belief prior is sufficiently inhibited. Three-year-olds' failures are limitations on their immature inhibitory abilities to overcome the rational prior.

At least two bodies of evidence support the continuing role of inhibitory executive functions in ToM reasoning. Inhibitory resources in older children remain insufficient to meet the increased demands of a false belief when combined with a desire to avoid (rather than to approach) the target of that belief (Freidman \& Leslie, 2004a, 2005; Leslie \& Polizzi, 1998; Leslie et al., 2005). Furthermore, when the inhibitory power is diminished, even adults may fail to predict actions based on others' false beliefs (LeBouc et al., 2012; German \& Hehman, 2006).

The ToMM-SP model also predicts that when the prior of choosing true belief is undermined, younger children would be more likely to succeed in reasoning about false belief. In the next section, we implement an extension of the model of the standard (HD) task evaluating data sets from the LD false belief task. The ability of the model to account for both of these contradictory patterns provides an important test.

\section{Increased uncertainty in "low-demand" false-belief task}

\section{Low-demand false-belief task}

While the characteristic developmental trajectory is that fouryear-olds are better at attributing false beliefs than three-yearolds, as discussed previously, younger children's performance improves under lower task demands. In such LD tasks, the salience of a child's own belief about the specific location of the target object might be undermined, as opposed to the standard false-belief task in which the child is relatively certain about the target's whereabouts (thus, HD). The increased uncertainty (or reduced confidence, used interchangeably in this paper) in their own belief facilitates the selection of a false belief in the LD tasks, while children's inhibitory power deployed in ToM reasoning is similar to that in the HD tasks. Using the Bayesian framework, we can estimate children's inhibitory power from their performance in the LD falsebelief task. Critically, we introduce a parameter to represent the child's uncertainty about his or her own belief (regarding the World); this works together with inhibition in reducing the priority of choosing the true belief (TB). The value of the increased uncertainty can be derived from the differences between children's performances in HD and LD false-belief tasks, such that we can have accurate estimation of the inhibitory power in the same fashion as in the model for HD task.

\section{ToMM+SP model for low-demand false-belief task}

To infer children's inhibitory power given their performance in the LD false-belief task, we introduced the uncertainty produced by the structure of the task, $\gamma_{W}$. Again, we assume an asymmetric beta prior on $\gamma_{W}$, with $\gamma_{W} \sim \operatorname{Beta}(5,16)$ for threeyear-olds and $\gamma_{W} \sim \operatorname{Beta}(2,16)$, such that $\gamma_{W}$ would have a mean around the empirical differences in the values between HD and LD tasks, 0.24 and $0.11^{2}$, for three-and four-yearolds, respectively, and still maintain small variances. Notice that when $\gamma_{W}$ is 0 , the model reverts to the one used for the standard false-belief task.

All other variables retain the notation outlined in the ToMM model for the standard false-belief task (see Fig. 4): the variable Desire $(D)$ has a large prior probability, $1-\varepsilon$, favoring the target object, with $\varepsilon \sim \operatorname{Beta}(1,10)$. The true belief content one attributes to Sally $(T B)$ is determined by one's own knowledge of the world, with probability $W-\gamma_{T B}$, and $\gamma_{T B} \sim$ Beta $(1,20)$. The child's confidence in the true belief is undermined in the LD scenarios, given by $W-\gamma_{T B}-\gamma_{W}$. The false-belief content $(F B)$ is determined by Sally's visual access to the world ( 0 , in a false-belief scenario), with the uncertainty about $F B$ given by the absolute value of $\left(W \cdot V-\gamma_{F B}\right)$, with $\gamma_{F B}$ $\sim$ Beta $(1,20)$.

As before, we assume that $I$ follows the Beta distribution with $I \sim \operatorname{Beta}(\alpha, \beta)$, where $\alpha \sim$ Uniform $(0,100)$ and $\beta \sim$ Uniform $(0,100)$ indicating no prior assumptions about the shape of the beta distribution of $I$ and to allow for a complete estimation of $I$ from the observed data.

The attributed belief is the most active belief between $F B$ and $T B$ with inhibition and reduced confidence. That is, the probability of $B$ is the larger one between the probability of the true belief with an inhibition and less confidence $\left(W-\gamma_{T B}-\right.$ $\gamma_{W}-I$ ) and the probability of the false belief (the absolute value of $\left.W \cdot V-\gamma_{F B}\right)$. After the selection, $B \sim \operatorname{Binomial}(1$, $\left.\max \left(F B, T B-\gamma_{W}-I\right)\right)$, the child attributes either the belief that there is no change $(B=0)$ or the belief that there is a change $(B$ $=1)$ to the agent. Attributed belief coupled with desire determines the child's action prediction $(A)$. In particular, when the attributed desire is to retrieve the object $(D=1)$, the child's

\footnotetext{
${ }^{2}$ Derived from empirical findings using low-demand false belief tasks, as summarized below.
} 


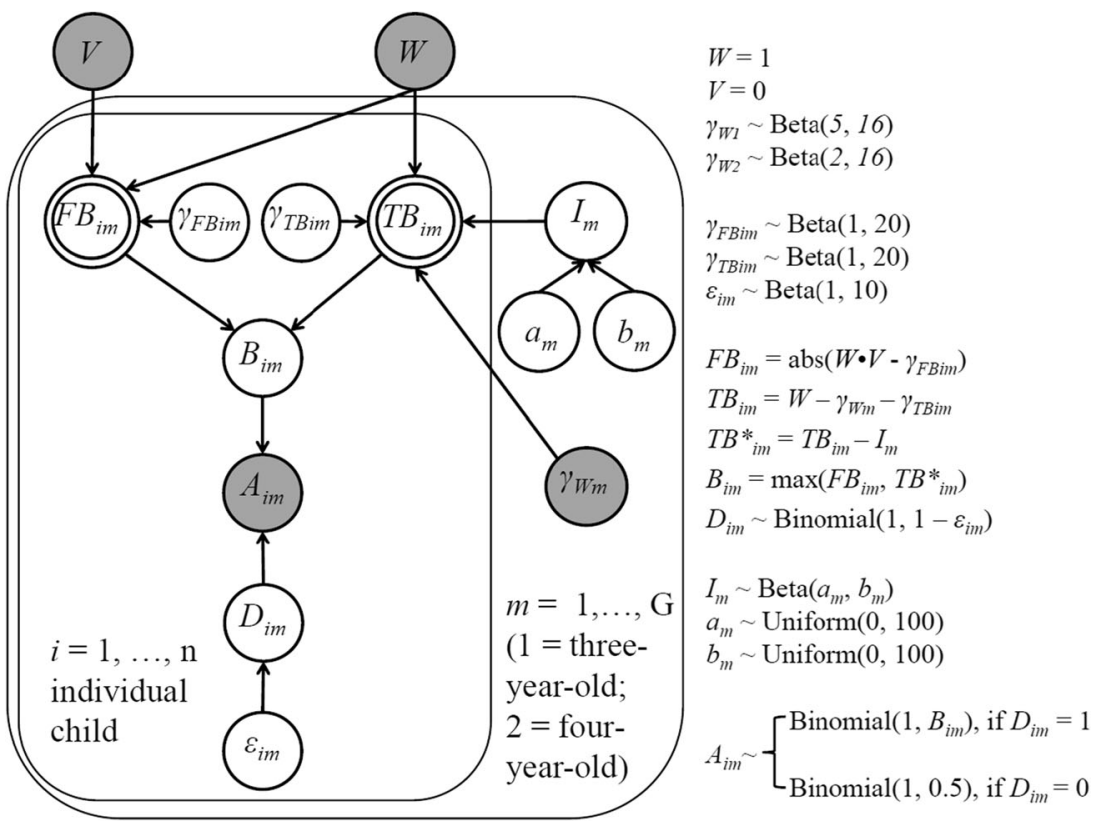

Fig. 4 The graphic model for ToMM + SP in LD false-belief task. W: world; $F B$ : false belief; $T B$ : true belief derived from one's own knowledge; $D$ : desire; $A$ : action; $V$ : visual access; $I$ : inhibition

prediction follows the attributed belief $(B)$ and $A \sim$ Binomial $(1, B)$; when the attributed desire is ambiguous $(D=0)$, the child's action prediction is random and $A \sim \operatorname{Binomial}(1,0.5)$. Table 3 gives the conditional probabilities amongst all variables.

\section{Bayesian inferences and model predictions}

\section{The data set}

Similar to the selection criterion for the standard (HD) falsebelief task, we constrained our search to studies that used the change-of-location task and reduced the child's confidence in his/her own belief about the target's whereabouts. Only studies conducted with children in Western cultures were considered. The selected data include five studies from five publications, including performances from 144 three-year-olds and 89 four-year-olds. See Table 4 for the list of selected publications and studies (the search of the studies was not intended to be exhaustive, but representative. As was the case for the HD model, to the best knowledge of the authors, this list includes all published data that met the selection criteria).

In all five studies using LD tasks, three-year-olds' were more likely to attribute false belief to others compared to their typical performances in HD tasks, while four-year-olds did not perform significantly better. Averaged across the five studies, three-year-olds were $24 \%$ more likely to attribute false belief to others in LD tasks compared to HD tasks, while four-yearolds were $11 \%$ more likely to do so (LD: $51 \%$ for three-yearolds, (s.d. $=0.158$ ); and $74 \%$ for four-year-olds, (s.d. $=0.065$ ); HD: $27 \%$ and $63 \%$ for three-and four-year-olds, respectively).
A Yates corrected $\chi^{2}$ analyses on observed performances in LD and HD false-belief tasks found a significant difference between three-year-olds' performances in LD-and HD falsebelief tasks $\left(\chi^{2}=25.96, p<.001\right)$, while there were no significant differences between four-year-olds' observed performances in LD and HD false belief tasks $\left(\chi^{2}=3.23, p>.05\right)$.

\section{Results}

The model was implemented using WinBUGS (Lunn, Thomas, Best, \& Spiegelhalter, 2000). Our results are based on drawing 3,000 samples from three separate chains with 300 burn-in periods $^{3}$ for each of the models. Convergence of the chains was assessed using the $\hat{R}$ statistic (Brooks \& Gelman, 1998).

Similar to the inferences based on the HD false-belief task, given children's action prediction in the LD false-belief task, we can reverse the causal chain and infer children's inhibitory power for different age groups. Figure 5 shows the posterior distribution of the inhibition parameter for each age group, estimated from the LD task, against the same estimation from HD task. It is clear that the posterior distribution of the inhibition parameter changes as a function of age group. The mean inhibitory power for three-year-olds is 0.25 (s.d. = $0.055,95 \%$ credible region is $0.131 \sim 0.349$ ), while the mean inhibitory power for four-year-olds is 0.59 (s.d. $=0.049,95 \%$ credible region is $0.496 \sim 0.686$ ). Importantly, the estimated inhibitory power given the LD false-belief task is similar to that based on the HD false-belief task, especially for four-

\footnotetext{
${ }_{3}^{3}$ Due to smaller samples in the LD tasks, more burn-in was required to reach a stable phase of estimation.
} 
Table 3 Conditional probability table for ToMM model in LD false-belief task

\begin{tabular}{llllllll}
\hline $\mathrm{P}(A=1 \mid B, D)$ & $B$ & $D$ & $\mathrm{P}\left(B=1 \mid F B, T B, I, W, V, \gamma_{T B}, \gamma_{F B}, \gamma_{W}\right)$ & $F B$ & $W$ & $V$ & $T B$ \\
\hline 1 & 1 & 1 & $\gamma$ & $\gamma_{F B}$ & 0 & 0 & $\gamma_{T B}$ \\
0 & 0 & 1 & $\gamma$ & $\gamma_{F B}$ & 0 & 1 & $\gamma_{T B}$ \\
0.5 & 1 & 0 & $1-\gamma$ & $1-\gamma_{W}-\gamma_{F B}$ & 1 & 1 & $1-\gamma_{W}-\gamma_{T B}$ \\
0.5 & 0 & 0 & $1-\gamma_{W}-\gamma_{T B}-I$ & $\gamma_{F B}$ & 1 & 0 \\
\hline
\end{tabular}

Note: $\mathbf{P}\left(\boldsymbol{B}=\mathbf{1} \mid \boldsymbol{F B}, \boldsymbol{T B}, \mathbf{I}, \boldsymbol{W}, \boldsymbol{V}, \boldsymbol{\gamma}_{\boldsymbol{T} \boldsymbol{B}}, \boldsymbol{\gamma}_{\boldsymbol{F B}}, \gamma_{W}\right)$ is the conditional probability of attributing a Belief $=1\left(B \sim \operatorname{Binomial}\left(1, \max \left(F B, T B-\gamma_{W}-I\right)\right)\right.$, max $(F B$, $\left.T B-\gamma_{W}-I\right)$ is the probability of $\mathrm{B}=1$ ) given the false belief, true belief, available inhibotory power, the real status of the world, the agent's visual access, and the noises of each belief

year-olds (in HD, estimated inhibitory power is 0.20 and 0.60 for three-and four-year-olds, respectively).

Figure 6 shows the posterior predictives for action prediction from the model. The mean posterior predictives are shown in black squares on the bar plot of observed data. The full posterior predictive distribution is shown in the left panel. It is clear that the model captures children's improved performance in the LD falsebelief task compared to that in the HD false-belief task, with higher success rates for both three-year-olds (mean: 0.55 , s.d. = 0.131 ) and four-year-olds (mean: 0.73, s.d. $=0.104$ ). Furthermore, comparing the posterior predictives of both LD and HD task ( 0.28 and 0.62 for three-and four-year-old, respectively), a three-year-old is $27 \%$ more likely to succeed in the LD task, and a four-year-old is $11 \%$ more likely to succeed, following a pattern similar to that of the observed data.

\section{Discussion}

We extended the model for the standard false-belief task to a LD task, in which the child is uncertain about his/her own belief, and evaluated the model's predictive power. Overall, the extended model provides an excellent qualitative fit for children's improved performances in the low-demand false-belief task.
Using a new dataset specific to the LD false-belief task, we replicate the range and difference in the estimated inhibitory power between age groups from the HD task, confirming inhibition as a viable mechanism in the selection process. It should be noted that there is a slight shift in the inferred inhibitory power for three-year-olds between the HD and LD tasks. This is at odds with the prediction of the model, since hypothetically, children of the same age should have similar inhibitory power regardless of the task's processing demands. There are several reasons why we might have observed this shift in our inference. First, the data set for the LD task is substantially smaller than for the HD task (with only 144 three-year-olds in the LD set vs. 389 in the HD set). Therefore, the estimation would be more variable for the LD set. In fact, variability in the inferred inhibition is much greater for the LD task. Second, and more interestingly, we might have underestimated the amount of uncertainty that the LD task induces in three-year-olds. Based on the empirical difference in performance we estimated $\gamma_{W}$ at 0.24 . As a check we also implemented the model assuming a greater uncertainty for three-yearsolds, with $\gamma_{W}$ beta( $(7,16)$, equivalent to a mean of 0.3 . With this higher uncertainty, estimated inhibition is now the same for the $\mathrm{HD}$ and LD task. However, given that we do not have a clear justification for this parameter value, we have chosen to present the full implementation of the model with the empirically derived

Table 4 Selected publications using low-demand change-of-location false-belief task

\begin{tabular}{|c|c|c|c|}
\hline Publication & Study/condition* & Age & Number of pass \\
\hline Bartsch, 1996 & $\begin{array}{l}\text { Study 1, OO and YY conditions: The character believed a desired object was in } \\
\text { one of two containers, while in fact it was in neither container }\end{array}$ & 3 & 10 out of 18 \\
\hline \multirow[t]{2}{*}{ Carlson, \& Moses, 2001} & \multirow{2}{*}{$\begin{array}{l}\text { Explicit false-belief task (with pictures): The child was told about the object's real } \\
\text { location, without seeing it }\end{array}$} & 3 & 21 out of 62 \\
\hline & & 4 & 31 out of 45 \\
\hline \multirow[t]{2}{*}{ Kikuno, Mitchell, \& Ziegler, 2007} & \multirow{2}{*}{$\begin{array}{l}\text { Study 3, aspect condition: In the character's absence, his Plasticine was reshaped } \\
\text { from a hat to an apple by his sister, and the child was asked where the main } \\
\text { character would search for his HAT (as opposed to his Plasticine; the former } \\
\text { was no longer existing, thus, less salient, while the latter was still present) }\end{array}$} & 3 & 16 out of 28 \\
\hline & & 4 & 22 out of 28 \\
\hline Wang, \& Leslie, in prep & $\begin{array}{l}\text { Low-demand condition: The target was removed from scene completely at the } \\
\text { character's absence }\end{array}$ & 3 & 15 out of 20 \\
\hline \multirow[t]{2}{*}{ Zaitchik, 1991} & \multirow{4}{*}{$\begin{array}{l}\text { Unseen condition: The child was told about the object's real location, without } \\
\text { seeing it }\end{array}$} & 3 & 11 out of 16 \\
\hline & & 4 & 13 out of 16 \\
\hline \multirow[t]{2}{*}{ Summary } & & 3 & 73 out of 144 \\
\hline & & 4 & 66 out of 89 \\
\hline
\end{tabular}




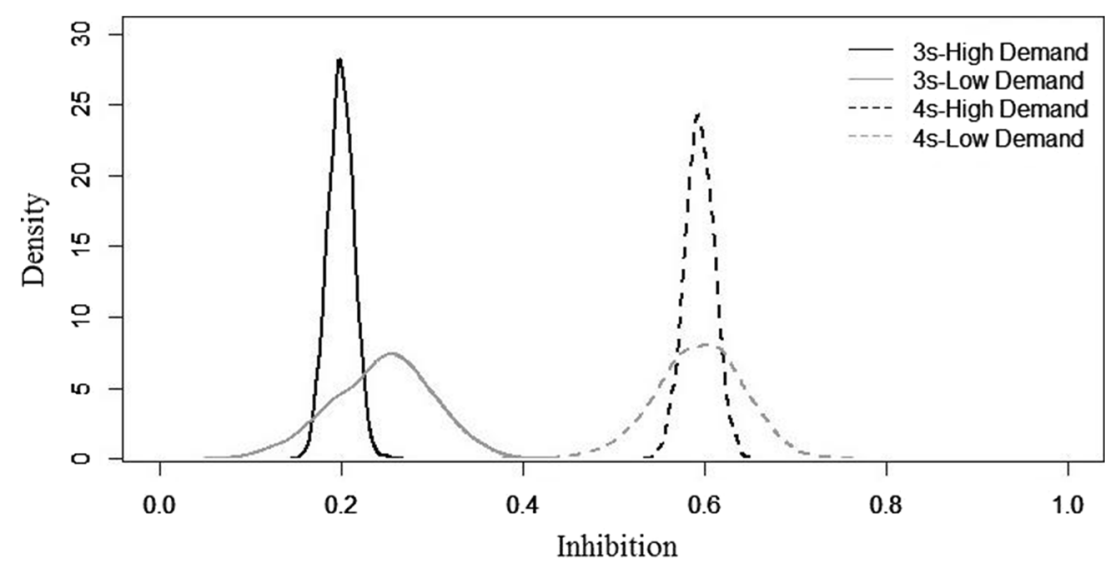

Fig. 5 Posterior distribution of inhibition ( $I$ ) based on LD and HD false-belief tasks, for each age group

values. A third possibility is that, in the extended model, the increased uncertainty interacts with the inhibitory power in reducing the chances of selecting the true belief. Therefore, the estimation of inhibitory power might be affected by the parameter of uncertainty. Given that the effect of reduced confidence in true belief is more prominent for three-year-olds (whose observed performances improve significantly from the standard HD to the LD false-belief task), the estimation of inhibitory power is more likely to be impacted. That is, the significant improvements in children's performances in the LD task is reflected in the estimation of inhibitory power for three-year-olds.

Nevertheless, the extended model captures the improvements in children's performances when the prior of choosing the true belief is undermined, for both three-and four-year-olds.

\section{General discussion}

We used a Bayesian framework to evaluate the role of inhibition in ToM reasoning and its development in three- and four- year-old children. We presented the first computational implementation of ToMM, modeling meta-data of the developmental shift from three-to four-years of age, and the facilitated performances in three-year-olds, that they are more likely to attribute false beliefs when their own belief of the world is uncertain. The strength of the ToMM+SP model is that it offers a unified theoretical mechanism underlying these developmental phenomena in ToM, as well as relevant empirical findings. The model provides excellent qualitative fit for children's performances in the standard false-belief task, and for the developmental shift between three- and four-year-olds. Using the same framework, we also modeled children's performance in a LD false-belief task, by quantitatively specifying the increased uncertainty in one's own belief. This is the first time this performance shift has been formally modeled. The extended model successfully captures children's facilitated performance in attributing false beliefs.

The ToMM+SP model assumes that we are fundamentally rational in belief-desire reasoning at all relevant ages and that children and adults use the same rational model in

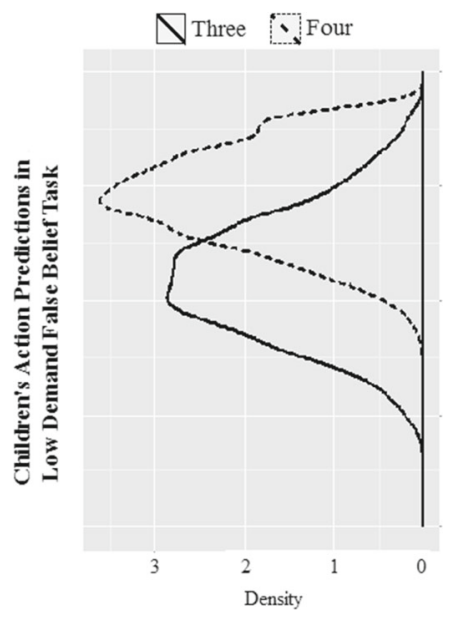

Fig. 6 Mean posterior predictives for action prediction in relation to the observed data (right panel) and the density of posterior predictives for action prediction (left panel) as a function of age group given the

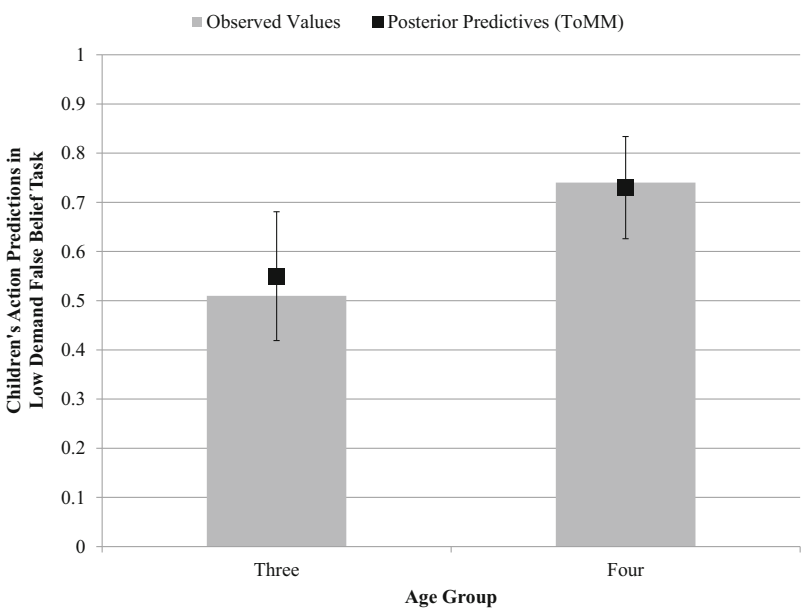

ToMM+SP model for the LD false-belief task. Error bars represent the standard error of the posterior predictive distributions 
representing others false belief. Under this hypothesis, threeyear-olds' failures are limitations on their immature inhibitory abilities. Both children and adults tend to attribute their own belief, typically true, to others. Therefore, one's own belief serves as the starting point for us to understand other perspectives (e.g., Tamir \& Mitchell, 2010). However, when the other person's belief is false (e.g., due to lack of access to the changes in the world), one needs to overcome his/her own true belief to correctly attribute a false belief. Children do not have sufficient power to overcome their own true belief when they are certain about it, at least in standard verbal false-belief tasks, until four years of age, giving rise to the developmental shift between three- and four-year-old children in ToM reasoning. However, when the demands of inhibiting our own true belief are lowered, for example, by low confidence in (high subjective uncertainty of) that belief, as in the low-demand falsebelief task, three-year-olds' capacity to represent others' false belief can be better revealed.

According to the ToMM+SP model, the key factor underlying the "three to four shift" in belief-desire reasoning is the role of inhibition. As specified in the Bayesian model implementing ToMM+SP, inhibition decreases the impact of (own) true belief, making it less likely to be selected for belief attribution. Consistent with the developmental trajectory in ToM reasoning, inhibition is a specific cognitive function that undergoes significant development from three to four years of age (Beck, Schaefer, Pang \& Carlson, 2011; Carlson, 2005; Diamond, Carlson \& Beck, 2005).

\section{Alternative explanations}

A prominent alternative hypothesis of ToM development is known as the conceptual shift theory, such as the theorytheory (Gopnik \& Wellman, 1992; Gopnik \& Wellman, 1994; also see Goodman et al. 2006), and the so-called "two-systems" accounts (Butterfill \& Apperly, 2013). Conceptual shift theory assumes that understanding of belief for three-year-olds (or, as addressed by the two-system accounts, infants and toddlers) is radically different from that of four-year-olds, and that the developmental shift reveals a conceptual revolution around the fourth birthday (Gopnik \& Wellman, 1992; Gopnik \& Wellman, 1994). Per the theorytheory, younger children's belief-desire reasoning is assumed to follow a simple model that a person's behavior is directly determined by the world, without considering other people's perceptual access (known as copy theory, CT; Wellman, 1990). However, this irrational model is assumed to fail in false-belief scenarios in which the person's belief is different from the world. The accumulation of evidence contradicting the early irrational model eventually forces children to abandon it and endorse the adult-like rational model that a person's belief is determined by that person's perceptual exposure to the environment (Perspective Theory, PT). After the more advanced PT model is favored, older children and adults no longer consider the $\mathrm{CT}$ model.

According to the theory-theory, the key factor underlying the developmental shift in ToM is "experience." Experience is a weighing factor and, importantly, has never been specified nor modeled as part of the computation of belief. By assuming that "experiences" select between the CT and PT models, theory-theory also begs the existence (or indeed possibility) of the rational model (PT) prior to any revision taking place. The mechanism of how children may construct the rational model from experiences is thus not explicitly instantiated in the Goodman et al. (2006) model. Rather, "experience" remains an assumption that is external to the model itself. By contrast, the key developmental factor in our model, inhibitory power, is captured within the model. Finally, children's facilitated performances in LD false-belief tasks pose a puzzle to theory-theory.

\section{The role of experience}

ToMM is best known as a modular cognitive system that computes others' mental states spontaneously (Leslie, 1994), and selects the appropriate belief to attribute with the help of inhibition. Here, we have focused on the role of inhibition in accounting for both the developmental trajectory and the effect of task demands in ToM, without directly accounting for the influence of experience. The development of all types of cognitive systems is likely to involve an interplay between brain maturation, change following exposure to the world including practice, and learning from evidence. Developing modular systems are no exception; for example, the acquisition of knowledge in module internal databases (Fodor, 1983, p. 81-82), and experience-dependent adjustment of prior likelihoods in visual modules (Scholl, 2005).

Therefore, while assuming a single rational model —in contrast to theory-theory's direct assumption of a transition from an irrational model to a rational model based on experienceToMM still acknowledges the importance of experience and practice (e.g., German \& Leslie, 2001; Roth \& Leslie, 1998). For example, the strength of the true-belief prior in the ToMM model might change in response to evidential experience (learning); the increasing power of inhibitory control might benefit from more practice or exercise of this ability, as Kovács (2009) argues happens in bilingual children as a result of inhibiting one language or another (for other examples of the benefits of practice see e.g., Diamond \& Lee, 2011; Setoh, Scott, \& Baillargeon, 2016; Spierer, Chavan, \& Manuel, 2013; cf. Thorell, Lindqvist, Bergman Nutley, Bohlin, \& Klingberg, 2009). According to the ToMM+SP model, these contributions of experience may make it more likely for children to consider another's false belief when it is in conflict 
with their own perspective, and therefore may contribute to the observed development from three- to four-years of age. ${ }^{4}$

\section{True-belief prior and infants' success in false-belief reasoning}

Finally, the ToMM+SP predicted rationality of spontaneous ToM reasoning throughout development (Leslie, 1987, 1994; Leslie \& Polizzi, 1998; Roth \& Leslie, 1998) has received support in recent years from studies showing that infants and toddlers can succeed with certain false-belief tasks. These are tasks often eliminate verbal content, or reduce it to a minimum, and use behavioral measures, such as violation-ofexpectation looking time, (e.g., Onishi \& Baillargeon, 2005; Scott, Baillargeon, Song, \& Leslie, 2010; Song, Onishi, Baillargeon, \& Fisher, 2008), anticipatory eye gaze (Southgate, Senju, \& Csibra, 2007; Wang \& Leslie, 2016), preferential looking (Scott, He, Baillargeon, \& Cummins, 2012), or spontaneous helping (Buttelmann, Carpenter, \& Tomasello, 2009; Southgate, Chevallier, \& Csibra, 2010).

Given infants' success in false-belief reasoning, it is natural to wonder if the true-belief prior does not exist in earlysuccess tasks. In other words, it is possible that only explicit verbal belief-related questions trigger the own true-belief prior on others' false belief, as a by-product of the standard falsebelief task itself, rather than the operational competence of the belief-desire reasoning system. This remains an open question

For example, Wang and Leslie (2016) used a non-verbal task and measured two-and half-year-old toddlers' anticipatory eye gaze. Toddlers could correctly anticipate the actor's search behavior when the actor had a true belief or when the toddlers did not know the final location of the object (LD task). When toddlers were certain about the object's final location, and therefore, had both a salient true-belief candidate and the actor's false-belief candidate were available for selection, their anticipation was split equally, revealing an unresolved decision. Schneider, Lam, Bayliss, and Dux (2012) obtained findings of split looking between the true-belief and false-belief candidates in adults in their "no-load condition.' In a looking-time study with infants, Yott and PoulinDubois (2012) found that the higher the infant scored in an executive function task, the longer the infant looked at the unexpected outcome in the false-belief task, suggesting again that the inhibitory demands of false belief may impact looking time measures.

However, a series of studies by Baillargeon and colleagues (Scott et al., 2010; Scott et al., 2012; Barrett et al., 2013; Setoh, Scott, \& Baillargeon, 2016) show that the basic Sally and Anne scenario can be presented verbally to toddlers who then show success on a number of measures. Especially relevant is Setoh, Scott, and Baillargeon (2016), which used not

\footnotetext{
${ }^{4}$ The authors thank Josh Tenenbaum for very useful discussion on this point.
}

only a verbal-narrative-plus-pictures method but also asked (verbal) direct prediction questions. A low-demand version of "Sally and Anne" found that 30-month-olds given two (non-ToM) practice questions with two possible answers were above chance on the subsequent belief question. Thirty-threemonth-olds needed slightly less practice in order to succeed in this task. These results support the existence of multiple factors in false-belief tasks among which are inhibition of own belief and selection of response to answer a direct question (Setoh et al., 2016; Scott \& Baillargeon, 2017). RubioFernandez \& Geurts (2016) takes a different tack suggesting, somewhat counterintuitively, that asking questions of the form, "Where... Sally...?", reorients attention away from Sally toward the actual location of the target. Presumably, attention to real location then increases the need for inhibition.

Roth and Leslie (1998:1) argued that to understand falsebelief development a task analysis that would "relate the structure of a given task to the underlying cognitive mechanisms that the task engages" is required (for each task). As tasks and measures multiply and the data becomes more complex, models of the relevant cognitive processes may need more parameters to account for more of the variance. What the studies in the present section have in common is the assumption that preschoolers already employ a concept of belief but over time become more adept at applying it accurately and robustly. Our aims in this paper have been to investigate how well we can model the " 3 to 4 shift" with a single performance parameter, Inhibition, and to quantify the changes in that parameter. The results support the idea that even a very simple model of Selection by Inhibition can indeed account not only for the classic shift but also for the difference between high- and low-demand tasks. We elaborate on this in the next section and point to future challenges.

\section{Model simplicity and extensions}

Whereas processing demands of false-belief tasks are multifold (Cohen \& German, 2009; Scott \& Baillargeon, 2017), we chose to model a small and restricted set of parameters. We were successful nevertheless in accounting for the main developmental shift in theory of mind. We used only the assumption of the ToMM that dual belief hypotheses, one based on actor's informational (e.g., visual) access to the world and another based on the child's own representation of that world, are generated spontaneously together with a process of selection, SP, that selects between them. One could put forward a more elaborate model of how the SP might operate. We chose instead to implement perhaps the simplest of assumptions: SP does no more than apply inhibition to the TB candidate, lowering its confidence level. We extended this to low-demand tasks simply by allowing inhibition power, $I$, to interact with the (subjective) confidence level of the world representation, $W$, that forms the basis of the TB candidate. We assume that 
any model of almost any kind of cognition will include "world" representations (what Leslie (1987) called "primary" representations) together with their confidence or noise level. Arguably, then, this assumption does not add to the complexity of our model; yet it allows us to capture the "low-demand" effect as a noisier representation of the target's final location.

We aimed here to quantify how well this simple model of selection would account for some of the main features of the ToM literature. Many questions remain, of course, any of which may require extensions to the model for false belief. For example, we did not use data from deceptive appearance tasks, such as "Smarties" (see p.14) though the effects in these tasks are much the same as in Sally and Anne. We conjecture that our model can extend straightforwardly to cover such tasks on the basis that (the child's) $W$ produces a change signal when the actual content of the container is revealed (raised to TB), whereas (the actor's) $V$ encodes access only to the initial state of the scenario in $W$ (raised to FB). Less straightforward, perhaps, are low-demand tasks such as "look first." Surian and Leslie (1999) suggested this minor change to the wording of the test question might increase the salience (reduce noise) of the false belief. It remains for future work to study whether incorporating increased competition from a less noisy FB candidate requires a more complex selection process than the very simple one we studied here. Other questions for further study include inferring pretense rather than belief (see Leslie 1994 for affinities and differences between these states); and inferring relations between belief and other mental states such as knowing and pretending (see German \& Leslie, 2001).

The relation between 'early' and 'later' belief tasks discussed above remains intriguing. Verbal questioning tasks may increase overall processing load, introduce pragmatic issues, increase the inhibitory demands of the own true belief, or indeed all of these (Setoh et al., 2016). Relative to standard false-belief tasks, basic data on "early" false belief remain sparse and indeed, with some replication failures, controversial. Until the field identifies more of the key experimental variables and practices relevant to reliable measurement, quantitative modeling may be premature. In any case, settling these questions is well beyond the scope of this paper; our hope is that the rational model presented here can refine these issues for further study.

Finally, decades of work on the " 3 to 4 shift" have relied entirely upon group-averaged data, as does the present model. Indeed, group-averaged data is enshrined in the very notion that younger preschoolers "fail" while older preschoolers "pass." However, such findings are irretrievably ambiguous. As Baker et al. (2016) point out, 70\% failure could be mean $70 \%$ of three's fail $100 \%$ of the time, or $100 \%$ pass $70 \%$ of the time, or any combination of probabilities as long as they average at or around $70 \%$ failure; likewise, with $70 \%$ passing for four's. Moreover, given that there is no such thing as a "group brain" wherein learning and development take place, the site of the shift must be the individual brain in the individual child. This simple fact dictates that we study the shift longitudinally within the individual child. This in turn requires novel statistical methods. Baker et al. (2016) developed such a method, called Bayesian change-point analysis. The authors gathered longitudinal data in the form of multiple single-case studies yielding individual cumulative records for 52 children followed for a year or more between three and five years old. Whereas some of these children showed the widely assumed floor to ceiling "sudden insight" shift, such children were a minority $(9.6 \%)$. Most children showed highly variable, indeed "stably unstable" records and many (44\%) provided evidence favoring no change (Bayesian methods quantify evidence for both $\mathrm{H}_{1}$ and $\mathrm{H}_{0}$ ). A major challenge for the present model will be to capture developmental change revealed, for the first time, by single-individual cumulative records.

\section{Summary}

Using a Bayesian framework, we adopted the ToMM+SP model to implement the critical role of inhibition in ToM reasoning, which can account for both the developmental shift from three to four years of age and the facilitated performances in three-year-olds when their own belief about the world is uncertain. We showed that Bayesian inference could provide insight into the detailed mechanisms of inhibition in the computation and development of ToM, as specified by the ToMM+SP model. This model explains how ToM reasoning can be rational (and thus the child be a rational learner) throughout development and yet still account for change. We quantitatively estimated children's changing inhibitory power, the specific cognitive resource that ToMM+SP assumes underlies this shift. In this way, we demonstrated both the relevance and benefits of Bayesian computational methods in advancing our understanding of ToM development. ToMM+ SP also has a wide empirical scope, accounting for both earlier and later developmental shifts and for the role of executive functions in ToM reasoning more generally.

While we have provided a unifying account of the developmental trajectory of ToM and the seemingly contradictory effect of task demand, important questions in ToM development remain. What is the role of experience? What is the nature, rate, and types of ToM learning trials "in the wild"? What does developmental change look like within the individual child who, unlike the averaged group, is the actual site of the computational mechanisms of learning (S. Baker, Leslie, Gallistel, \& Hood 2016)? Assumed to play a central role, yet almost completely unstudied, the nature of experience in change is mostly a matter of speculation. We urge renewed focus on these questions. 
Acknowledgements We are grateful to Josh Tenenbaum for useful discussion on the theory-theory, to Randy Gallistel and Vicky Froyen for helpful comments and suggestions on model comparison, and to Jessica Cantlon and two anonymous reviewers for insightful and constructive comments on an earlier draft. This research was supported by NSF grant BCS-0922184 to A.M.L.

\section{Compliance with ethical standards}

Conflicts of interest The authors have no conflicts of interest.

\section{References}

Apperly, I. A., Samson, D., Chiavarino, C., \& Humphreys, G. W. (2004). Frontal and temporo-parietal lobe contributions to theory of mind: neuropsychological evidence from a false-belief task with reduced language and executive demands. Journal of Cognitive Neuroscience, 16(10), 1773-1784.

Baillargeon, R., Scott, R. M., \& He Z. (2010). False-belief understanding in infants. Trends in Cognitive Sciences, 14, 110-118.

Baker, C. L., Goodman, N. D., \& Tenenbaum, J. B. (2008). Theory-based social goal inference. Proceedings of the thirtieth annual conference of the cognitive science society.

Baker, C. L., Saxe, R., \& Tenenbaum, J. B. (2009). Action understanding as inverse planning. Cognition, 113(3), 329-349.

Baker, C. L., Saxe, R. R., \& Tenenbaum, J. B. (2011). Bayesian theory of mind: Modeling joint belief-desire attribution. Proceedings of the thirty-second annual conference of the cognitive science society.

Baker, S. T., Leslie, A. M., Gallistel, C. R., \& Hood, B. M. (2016). Bayesian change-point analysis reveals developmental change in a classic theory of mind task. Cognitive Psychology, 91, 124-149.

Baron-Cohen, S., Leslie, A. M., \& Frith, U. (1985). Does the autistic child have a "theory of mind"? Cognition, 21, 37-46.

Barrett, H.C., Broesch, T., Scott, R.M., He, Z., Baillargeon, R., Wu, D., Bolz, M., Henrich, J., Setoh, P., Wang, J., \& Laurence, S. (2013). Early false-belief understanding in traditional non-Western societies Proceedings of the Royal Society B: Biological Science, 280 (1755), 20122654.

Bartsch, K. (1996). Between desires and beliefs: Young children's action predictions. Child Development, 67, 1671-1685.

Beck, D. M., Schaefer, C., Pang, K., \& Carlson, S. M. (2011). Executive Function in Preschool Children: Test-Retest Reliability. Journal of Cognition and Development, 12(2), 169-193.

Berthiaume, V. G., Shultz, T. R., \& Onishi, K. H. (2013). A constructivist connectionist model of transitions on false-belief tasks. Cognition, $126(3), 441-458$

Brooks, S. P., \& Gelman, A. (1998). General methods for monitoring convergence of iterative simulations. Journal of Computational and Graphical Statistics, 7(4), 434-455.

Buttelmann, D., Carpenter, M., \& Tomasello, M. (2009). Eighteenmonth-old infants show false belief understanding in an active helping paradigm. Cognition, 112(2), 337-342.

Butterfill, S. A., \& Apperly, I. A. (2013). How to construct a minimal theory of mind. Mind \& Language, 28(5), pp. 606-637

Callaghan, T., Rochat, P., Lillard, A., Claux, M. L., Odden, H., Itakura, S., Singh, S. (2005). Synchrony in the onset of mental-state reasoning evidence from five cultures. Psychological Science, 16(5), 378-384.

Carlson, S., \& Moses, L. J. (2001). Individual differences in inhibitory control and children's theory of mind. Child Development, 72(4), 1032-1053

Carlson, S. M. (2005). Developmentally sensitive measures of executive function in preschool children. Developmental Neuropsychology, 28(2), 595-616.
Carlson, S. M., Moses, L. J., \& Hix, H. R. (1998). The role of inhibitory processes in young children's difficulties with deception and false belief. Child Development, 69(3), 672-691.

Carpenter, M., Call, J., \& Tomasello, M. (2002). A new false belief test for 36-month-olds. British Journal of Developmental Psychology, 20, 393-420.

Cassidy, K. W. (1998). Three-and four-year-old children's ability to use desire-and belief-based reasoning. Cognition, 66(1), B1-B11.

Chandler, M., Fritz, A. S., \& Hala, S. (1989). Small-scale deceit: Deception as a marker of two-, three-, and four-year-olds' early theories of mind. Child Development, 1263-1277.

Clements, W. A., Rustin, C. \& McCallum, S. (2000). Promoting the transition from implicit to explicit understanding: A training study of false belief. Developmental Science, 3,81-92, https://doi.org/10. 1111/1467-7687.00102

Cohen, A.S., \& German, T. (2009): Encoding of others' beliefs without overt instruction. Cognition, 111, 356-363.

Dennett, D. C. (1989). The international stance. Cambridge: The MIT press.

Diamond, A., Carlson, S. M., \& Beck, D. M. (2005). Preschool children's performance in task switching on the dimensional change card sort task: separating the dimensions aids the ability to switch. Developmental Neuropsychology, 28(2), 689-729.

Diamond, A., \& Lee, K. (2011). Interventions shown to aid executive function development in children 4 to 12 years old. Science, 333(6045), 959-964.

Fodor, J. A. (1983). The Modularity of Mind. Cambridge: MIT Press.

Friedman, O., \& Leslie, A. M. (2004a). A developmental shift in processes underlying successful belief-desire reasoning. Cognitive Science, 28(6), 963-977.

Friedman, O., \& Leslie, A. M. (2004b). Mechanisms of belief-desire reasoning. Inhibition and bias. Psychological Science, 15(8), 547552.

Friedman, O., \& Leslie, A.M. (2005). Processing demands in beliefdesire reasoning: inhibition or general difficulty? Developmental Science, 8, 218-225.

German, T. P., \& Hehman, J. A. (2006). Representational and executive selection resources in 'theory of mind': evidence from compromised belief-desire reasoning in old age. Cognition, 101(1), 129-152.

German, T. P., \& Leslie, A. M. (2001). Children's inferences from 'knowing'to 'pretending' and 'believing'. British Journal of Developmental Psychology, 19(1), 59-83.

Goodman, N. D., Baker, C. L., Bonawitz, E. B., Mansinghka, V. K., Gopnik, A., Wellman, H., \& Tenenbaum, J. B. (2006). Intuitive theories of mind: A rational approach to false belief. Proceedings of the twenty-eighth annual conference of the cognitive science society.

Gopnik, A., \& Astington, J. W. (1988). Children's understanding of representational change and its relation to the understanding of false belief and the appearance-reality distinction. Child Development, 59(1), 26-37.

Gopnik, A., \& Wellman, H. (1992). Why the child's theory of mind really is a theory of mind. Mind \& Language, 7, 145-171.

Gopnik, A., \& Wellman, H. (1994). The theory theory. In L. A. Hirschfeld \& S. A. Gelman (Eds.), Mapping the mind: Domain specificity in cognition and culture (pp. 257-293). New York: Cambridge University Press.

Hartwright, C. E., Apperly, I. A., \& Hansen, P. C. (2012). Multiple roles for executive control in belief-desire reasoning: distinct neural networks are recruited for self perspective inhibition and complexity of reasoning. Neuroimage, 61(4), 921-930.

Hickling, A. K., Wellman, H. M., \& Gottfried, G. M. (1997). Preschoolers' understanding of others' mental attitudes towards pretend happenings. British Journal of Developmental Psychology, 15(3), 339-354. 
Kikuno, H., Mitchell, P., \& Ziegler, F. (2007). How do young children process beliefs about beliefs? Evidence from response latency Mind \& Language, 22(3), 297-316.

Koós, O., Gergely, G., Csibra, G., \& Bíró, S. (1997). Why eating Smarties makes you smart. Understanding false belief at the age of 3 . Poster presented at the biennial meeting of the Society for Research in Child Development, Washington, DC.

Kovács, A.M. (2009). Early bilingualism enhances mechanisms of falsebelief reasoning. Developmental Science, 12, 48-54.

LeBouc, R., Lenfant, P., Delbeuck, X., Ravasi, L., Lebert, F., Semah, F., \& Pasquier, F. (2012). My belief or yours? Differential theory of mind deficits in frontotemporal dementia and Alzheimer's disease. Brain, 135, 3026-3038.

Leslie, A. M. (1987). Pretense and representation: The origins of "theory of mind". Psychological Review, 412-426.

Leslie, A. M. (1994). Pretending and believing: issues in the theory of ToMM. Cognition, 50(1-3), 211-238.

Leslie, A. M., Friedman, O., \& German, T. P. (2004). Core mechanisms in "theory of mind". Trends in Cognitive Science, 8(12), 528-533.

Leslie, A. M., German, T. P., \& Polizzi, P. (2005). Belief-desire reasoning as a process of selection. Cognitive Psychology, 50(1), 45-85.

Leslie, A. M., \& Polizzi, P. (1998). Inhibitory processing in the false belief tasks: Two conjectures. Developmental Science, 247-253.

Leslie, A. M., \& Thaiss, L. (1992). Domain specificity in conceptual development: neuropsychological evidence from autism. Cognition, 43(3), 225-251.

Liu, D., Wellman, H. M., Tardif, T., \& Sabbagh, M. A. (2008). Theory of mind development in Chinese children: a meta-analysis of falsebelief understanding across cultures and languages. Developmental Psychology, 44(2), 523-531.

Lunn, D. J., Thomas, A., Best, N., \& Spiegelhalter, D. (2000). WinBUGS-a Bayesian modelling framework: concepts, structure, and extensibility. Statistics and computing, 10(4), 325-337.

Matsui, T., Rakoczy, H., Miura, Y., \& Tomasello, M. (2009). Understanding of speaker certainty and false-belief reasoning: a comparison of Japanese and German preschoolers. Developmental Science, 12(4), 602-613.

McKinnon M.C., \& Moscovitch, M. (2007). Domain-general contributions to social reasoning: theory of mind and deontic reason-ing reexplored. Cognition, 102, 179-218.

Mitchell, P., \& Lacohée, H. (1991). Children's early understanding of false belief. Cognition, 107-127.

Ntzoufras, I. (2009). Bayesian modeling using WinBUGS. New York: Wiley

Onishi, K. H., \& Baillargeon, R. (2005). Do 15-month-old infants understand false beliefs? Science, 308(5719), 255-258.

Perner, J., Leekam, S. R., \& Wimmer, H. (1987). Three-year-olds' difficulty with false belief: The case for a conceptual deficit. British Journal of Developmental Psychology, 5(2), 125-137.

Peterson, C. C., \& Siegal, M. (1999). Representing inner worlds: Theory of mind in autistic, deaf, and normal hearing children. Psychological Science, 10(2), 126-129.

Richardson, H. L., Baker, C. L., Tenenbaum, J. B., \& Saxe, R. R. (2012). The development of joint belief-desire inferences. Proceedings of the 34th annual meeting of the cognitive science society.

Riggs, K. J., Peterson, D. M., Robinson, E. J., \& Mitchell, P. (1998). Are errors in false belief tasks symptomatic of a broader difficulty with counterfactuality? Cognitive Development, 13(1), 73-90.

Riggs, K. J., \& Simpson, A. (2005). Young children have difficulty ascribing true beliefs. Developmental Science, 8(3), F27-F30.

Robinson, E. J., \& Mitchell, P. (1995). Masking of children's early understanding of the representational mind: Backwards explanation versus prediction. Child Development, 1022-1039.

Roth, D., \& Leslie, A. M. (1998). Solving belief problems: toward a task analysis. Cognition, 66(1), 1-31.
Rubio-Fernández, P., \& Geurts, B. (2016). Don’t Mention the Marble! The Role of Attentional Processes in False-Belief Tasks. Review of Philosophy and Psychology, 7(4), 835-850. https://doi.org/10.1007/ s13164-015-0290-z

Russell, J. (1996). Agency: Its Role in Mental Development (pp. 180242). Hove: Erlbaum.

Russell, J., Mauthner, N., Sharpe, S., \& Tidswell, T. (1991). The 'windows task' as a mea-sure of strategic deception in preschoolers and autistic subjects. British Journal of Developmental Psychology, 9, 331-349.

Sabbagh, M. A., Bowman, L. C., Evraire, L. E., \& Ito, J. M. (2009). Neurodevelopmental correlates of theory of mind in preschool children. Child Development, 80(4), 1147-1162.

Samson, D., Apperly, I. A., Kathirgamanathan, U., \& Humphreys, G. W. (2005). Seeing it my way: a case of a selective deficit in inhibiting self-perspective. Brain, 128, 1102-1111.

Saxe, R., Schulz, L. E., \& Jiang, Y. V. (2006). Reading minds versus following rules: dissociating theory of mind and executive control in the brain. Social Neuroscience, 1(3-4), 284-298.

Schneider, D., Lam, R., Bayliss, A., \& Dux, P. (2012). Cognitive load disrupts implicit theory-of-mind processing. Psychological Science, 23(8), 842-7.

Scholl, B. J. (2005). Innateness and (Bayesian) visual perception. In P. Carruthers, S. Laurence, \& S. Stich (Eds.), The innate mind: Structure and contents (pp. 34-52). Oxford University Press.

Scholl, B. J., \& Leslie, A. M. (2001). Minds, modules, and meta-analysis. Child Development, 72(3), 696-701.

Scott, R. M., \& Baillargeon, R. (2017). Early False-Belief Understanding. Trends in Cognitive Sciences, 21(4), 237-249.

Scott, R.M., Baillargeon, R., Song, H., \& Leslie, A.M. (2010). Attributing false beliefs about non-obvious properties at 18 months. Cognitive Psychology, 61, 366-395.

Scott, R.M., He, Z., Baillargeon, R., Cummins, D. (2012). False-belief under-standing in 2.5-month-olds: Evidence from two novel verbal spontaneous-response tasks. Developmental Science, 15, 181-193.

Setoh, P., Scott, R.M., \& Baillargeon, R. (2016). Two-and-a-half-yearolds succeed at a traditional false-belief task with reduced processing demands. PNAS, 113, 13360-13365.

Song, H., Onishi, K., Baillargeon, R., \& Fisher, C. (2008). Can an agent's false belief be corrected through an appropriate communication? Psychological reasoning in 18-month-old infants. Cognition, 109, 295-315.

Southgate, V., Chevallier, C., \& Csibra, G. (2010). Seventeen-month-olds appeal to false beliefs to interpret others' referential communication. Developmental Science, 13(6), 907-912.

Southgate, V., Senju, A., \& Csibra, G. (2007). Action anticipation through attribution of false belief by 2-year-olds. Psychological Science, 18(7), 587-592.

Spierer, L., Chavan, C. F., \& Manuel, A. L. (2013). Training-induced behavioral and brain plasticity in inhibitory control. Frontiers in human neuroscience, 7, 427.

Surian, L., \& Leslie, A. M. (1999). Competence and performance in false belief understanding: A comparison of autistic and normal 3-yearold children. British Journal of Developmental Psychology, 17, 141155.

Tamir, D. I., \& Mitchell, J. P. 2010: Neural correlates of anchoring-andadjustment during mentalizing Proceedings of the National Academy of Sciences, 107(24), 10827-10832.

Thorell, L. B., Lindqvist, S., Bergman Nutley, S., Bohlin, G., \& Klingberg, T. (2009). Training and transfer effects of executive functions in preschool children. Developmental Science, 12(1), 106-113.

van der Meer, L., Groenewold, N. A., Nolen, W. A., Pijnenborg, M., \& Aleman, A. (2011). Inhibit yourself and understand the other: neural basis of distinct processes underlying Theory of Mind. Neuroimage, 56(4), 2364-2374. 
Wang, L., \& Leslie, A. M. (2016). Is implicit theory of mind the 'real deal'? The own-belief/true-belief default in adults and young preschoolers. Mind \& Language, 31(2), 147-176.

Wellman, H. M. (1990). The Child's Theory of Mind. Cambridge: The MIT Press.

Wellman, H. M., \& Bartsch, K. (1988). Young children's reasoning about beliefs. Cognition, 30, 239-277.

Wellman, H. M., Cross, D., \& Watson, J. (2001). Meta-analysis of theoryof-mind development: The truth about false belief. Child Development, 72(3), 655-684.
Wimmer, H., \& Hartl, M. (1991). Against the Cartesian view of mind: Young children's difficulty with own false beliefs. British Journal of Developmental Psychology, 9, 125-138.

Wimmer, H., \& Weichbold, V. (1994). Children's theory of mind: Fodor's heuristics examined. Cognition, 53(1), 45-57.

Yott, J., \& Poulin-Dubois, D. (2012). Breaking the rules: do infants have a true understanding of false belief? British Journal of Developmental Psychology, 30, 156-171.

Zaitchik, D. (1991). Is only seeing really believing? Sources of the true belief in false belief task. Cognitive Development, 6, 91-103. 